\title{
Polarization-resolved terahertz imaging of hybrid fiber-reinforced composite laminate subject to low-velocity impact
}

\author{
Junliang Dong, Alexandre Locquet, D. S. Citrin \\ Georgia Tech-CNRS UMI2958, Georgia Tech Lorraine, 2 Rue Marconi, 57070 Metz, France \\ School of Electrical and Computer Engineering, Georgia Institute of Technology, Atlanta, GA 30332, USA \\ junliang.dong@gatech.edu,alexandre@gatech.edu,david.citrin@ece.gatech.edu
}

\begin{abstract}
By taking advantage of the interaction between terahertz polarization and carbonfiber orientation, impact-induced intra- and inter-laminar damages at the same interface are differentiated via terahertz polarization-resolved imaging in three dimensions.
\end{abstract}

OCIS codes: (110.6795) Terahertz imaging, (120.4290) Nondestructive testing.

\section{Introduction}

Hybrid fiber-reinforced composites, which combine two or more types for fibers in the same composites in order to benefit from the merits of each constituent fiber type, have been used in many applications which need to meet both the requirements of cost and performance. However, fiber-reinforced composite laminates are vulnerable to impact. The resulting impact-induced damage in laminates involves a combination of intra-laminar damage (matrix cracking and fiber/matrix debonding) and inter-laminar damage (delaminations). Various NDE techniques, such as ultrasonic $\mathrm{C}$-scans, eddy current, thermography and X-ray CT, have been developed for impact damage detection in composite structures over the last few decades. However, not all these methods can provide information in depth to observe the morphology and evolution of the impact damage throughout the thickness of the laminate [1]. Terahertz(THz) imaging, which can provide a non-invasive, noncontact, and nonionizing method to characterize composites, is a promising modality for nondestructive evaluation of fiber composites. In this study, THz imaging is firstly applied to a hybrid fiber-reinforced composite laminate, comprised of unidirectional glass/epoxy and carbon/epoxy laminae with a crossply stack pattern.By taking advantage of the sensitivity of the THz polarization and carbon-fiber orientation, matrix cracking and delamination at the same interface can be differentiated. $\mathrm{THz} \mathrm{C}$ - and B-scans exhibit the propagation of the impact damage from the top to the bottom surface in three dimensions.

\section{Principle}

For unidirectional carbon fiber-reinforced composites, the conductivity is anisotropic and depends on the THz polarization and fiber orientation, which can be expressed as

$$
\sigma(\theta)=\sigma_{l} \cos ^{2} \theta+\sigma_{t} \sin ^{2} \theta
$$

where $\theta$ is the angle between the THz polarization and fiber orientation and $\sigma_{l}$ and $\sigma_{t}$ are the longitudinal and transverse conductivities, respectively. Along the fiber direction, the electric current flows through the carbon fibers, so the longitudinal conductivity depends on the conductivity of carbon fibers and on the fiber volume fraction. For the transverse conductivity, because the resin material is nonconductive, the flow of electric current only occurs due to random contact between adjacent carbon fibers, which depends on the manufacturing process and the quality of the composites [2]. Therefore, the longitudinal conductivity is much higher than the transverse conductivity.

By analyzing the electric conductivity, we find out that (1) when the THz polarization is parallel to the orientation of carbon fibers, the electric conductivity is maximum and the THz reflectivity also reaches the maximum; (2) when the $\mathrm{THz}$ polarization is perpendicular to the carbon fibers, conductivity and reflectivity achieve their minimum. Therefore, monitoring the reflection coefficient across the surface of carbon fiber composites with $\mathrm{THz}$ imaging can be utilized as a method to characterize the impact damage on the surface [3]. When carbon fiber-reinforced composites suffer from impact damage, carbon fibers will be distorted in the damage area, which will lead to spatial variation of the reflection coefficient as well as the polarization anisotropy. The reflection coefficient in regions with and without impact damage can be more easily distinguished with polarization parallel to the carbon-fiber orientation. 


\section{Experiment and Results}

$\mathrm{THz}$ C-scans at the interface between the glass/epoxy and carbon/epoxy show quite different damage patterns for the two polarizations, shown in Fig. 1. For parallel polarization, the damaged region shows lower contrast due to the existence of matrix cracking and fiber distortion. However, for perpendicular polarization, the damaged region shows higher contrast. This higher contrast indicates the existence of an air gap originating in the separation of the carbon/epoxy and glass/epoxy plies, i.e., delamination.
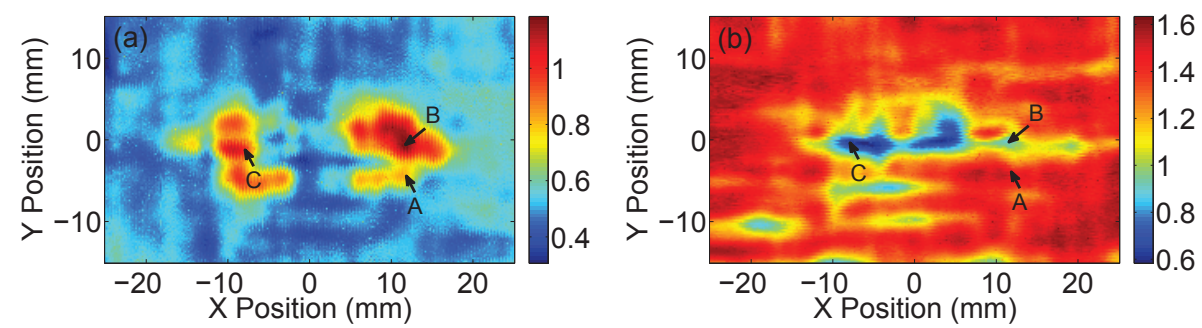

Fig. 1. THz C-scans at the interface between the glass/epoxy and carbon/epoxy with polarization perpendicular (a) and parallel (b) to the carbon fiber orientation.
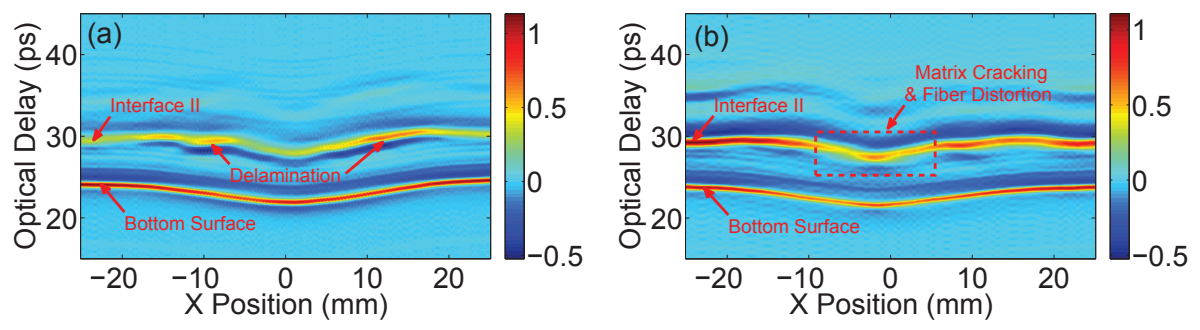

Fig. 2. B-scans incident from the bottom surface (along section $y=0$ ) and with polarization perpendicular (a) and parallel (b) to the carbon-fiber orientation.

The change of the contrast in the damaged region mainly depends on two factors: (1) the thickness of the delamination (a narrower air gap leads to a smaller difference due to partial cancellation of the negative and positive peaks) and (2) the reflection coefficient (impact-induced matrix cracking and fiber distortion in the carbon/epoxy will lower the value of the reflection coefficient). Therefore, for C-scans with perpendicular polarization, the thickness of the delamination dominates the change of the contrast, and the C-scans reveal the delamination area at the interface. For $\mathrm{C}$-scans with parallel polarization, the reflection coefficient at the interface dominates the change of the contrast.

B-scans obtained incident from the bottom surface are shown in Fig. 2. Typical damage features can be observed in depth, including surface bending, delamination, matrix cracking and fiber distortion. Therefore, the total set of Cand B-scans exhibits the evolution of the impact-induced damage from the surface to the internal interface in three dimensions.

\section{References}

1. Dong J, Kim B, Locquet A, McKeon P, Declercq N, Citrin DS. Nondestructive evaluation of forced delamination in glass fiber-reinforced composites by terahertz and ultrasonic waves. Composites Part B: Engineering. 2015;79(0):667-75.

2. Athanasopoulos N, Kostopoulos V. Prediction and experimental validation of the electrical conductivity of dry carbon fiber unidirectional layers. Composites Part B: Engineering. 2011;42(6):1578-87.

3. Yang S-H, Kim K-B, Oh HG, Kang J-S. Non-contact detection of impact damage in CFRP composites using millimeter-wave reflection and considering carbon fiber direction. NDT \& E International. 2013;57(0):45-51. 